\title{
Es geht um Finanzierung, nicht um Kompetenzen
}

\author{
Im Zusammenhang mit der parlamentarischen Initiative «Gesetzliche Anerkennung \\ der Verantwortung der Pflege» sorgen diverse Missverständnisse und, wie es zur \\ Politik wohl zu gehören scheint, auch falsche Behauptungen für Verwirrung. Zeit \\ für eine Klärung aus juristischer Sicht.
}

Pierre-André Wagner

Rechtsanwalt, LL.M. dipl. Pflegefachmann AKP, Leiter Rechtsdienst des Schweizer Berufsverbandes der Pflegefachfrauen und Pflegefachmänner SBK

Eine Stellungnahme der FMH findet sich im Anschluss auf S. 1097. 1 www.parlament.ch/d/suche/
seiten/geschaefte.aspx? gesch_id=20110418
Die erste Frage, die diese Verwirrung zum Ausdruck bringt: Würden mit der Initiative die Grenzen zwischen Medizin und Pflege neu gezogen? Und daraus abgeleitet: Würden den Pflegefachpersonen Kompetenzen zugeschlagen, die bis anhin den Ärzten und Ärztinnen zustehen - mit unabsehbaren haftungsrechtlichen Konsequenzen?

Die kurze Antwort lautet: Nein, nicht im Mindesten. Denn die Initiative bezieht sich auf das KVG ein Gesetz, das keine Haftungsfragen regelt.

Aber sie bringt - auch hinsichtlich der Haftung eine überfällige Klärung, die sowohl den Ärzten als auch den Pflegenden zugute kommt.

Mit anderen Worten geht es ausschliesslich, kurz und präzise darum, dass Leistungen, die Pflegefachpersonen schon jetzt in voller eigener (rechtlicher und fachlicher) Verantwortung, also ohne ärztlichen Auftrag bzw. ärztliches Visum, erbringen dürfen, ebenfalls ohne ärztliches Visum von der Krankenversicherung übernommen werden. welcher Leistungserbringer unter welchen Voraussetzungen von den Krankenkassen bezahlt werden müssen.

Damit ist der Schlüssel zum Verständnis der Initiative eine Eigenart des Pflegeberufes, die den meisten Nicht-Berufsangehörigen - einschliesslich der meisten Politiker - unbekannt ist: nämlich der Umstand, dass die Pflege aus zwei Bereichen besteht. Im einen sind die Pflegefachpersonen diagnostisch und therapeutisch tätig. Diese Verrichtungen gehören zu den Kernkompetenzen der Ärztinnen und Ärzte, weshalb dies ausschliesslich auf deren Delegation hin geschieht. Fatalerweise reduzieren althergebrachte Stereotypen die Pflege auf diesen einen Bereich - die Pflegende als «Hilfskraft» des Arztes ein Zerrbild, dem auch das KVG huldigt.

\section{Überforderte Ärzte}

Dabei wird der zweite Bereich der Pflege übersehen, in dem sie eigenständig, autonom und - nicht zu-

\section{«Es geht nicht um eine Aufwertung der Pflege, sondern um die Anerkennung ihrer Verantwortung.»}

\section{Zerrbild der Pflege}

Der etwas sperrige Name der Initiative wurde mit Bedacht gewählt: Es geht nicht um eine Aufwertung der Pflege, sondern um die Anerkennung ihrer Verantwortung. Anerkennen kann man nur Vorbestehendes. Die Initiative will, dass die Verantwortung, die die Pflegenden bereits tragen, endlich auch im KVG anerkannt wird.

Es sei daran erinnert, dass das KVG keine Kompetenzen regelt. Es sagt nicht, welche Gesundheitsberufe wofür zuständig sind und wer was tun darf. Was zu den Aufgaben und zur Verantwortung der Pflegefachpersonen gehört, legen vielmehr die kantonalen Gesundheitsgesetze fest. Das KVG regelt einzig die Finanzierung: Es legt fest, welche Leistungen letzt - eigenverantwortlich handelt. Dieser Bereich, zu dem unter anderem die Abklärung des Pflegebedarfs, die Beratung des Patienten und seines Umfeldes und vieles mehr gehört, wird vom sog. Pflegeprozess gesteuert und liegt fernab jeder ärztlichen Kompetenz. Die gesetzliche Grundlage für den eigenverantwortlichen Bereich der Pflege befindet sich, wie bereits erwähnt, im kantonalen Gesundheitsrecht - zum Teil ganz explizit. Das sieht, zum Beispiel in Solothurn, so aus:

$\ll^{2}$ Zur Berufsausübung in eigener Verantwortung gehören die Planung und Ausführung der Pflege sowie die Evaluierung deren Wirksamkeit. Sie umfasst insbesondere die Feststellung der Bedürfnisse des Patienten oder der 
Patientin, die vorübergehende oder dauernde Unterstützung bei der Ausübung der Lebensaktivitäten und Präventionsmassnahmen sowie die Information und Beratung.

${ }^{3}$ Zum delegierten Aufgabenbereich gehört die Ausführung der schriftlichen Verordnungen der verantwortlichen Arztperson betreffend Prävention, medizinische Diagnose und Behandlung» [2].

$2 \S 45$ Abs. 2 und 3 der Vollzugsverordnung zum Gesundheitsgesetz vom 28.6. 1999.

3 Sie könnte sich einzig damit entlasten, der delegierende Arzt habe Delegationsgrundsätze verletzt, z. B. indem er sie ungenügend instruiert, beaufsichtigt oder kontrolliert habe, bzw. sie sei aufgrund ihrer Ausbildung und Lage gewesen, die Risiken der delegierten Tätigkeit richtig einzuschätzen.

4 Vgl. das Referat von Annette Jamieson (Gesundheitspolitik Helsana) anlässlich des Treffens der parlamentarischen Gruppe Pflege vom 12. März 2014.

5 Vgl. Leserbrief von Dr. Severin Lüscher in der Schweizerischen Ärztezeitung 2014/11

6 Vgl. Pierre-André Wagner, «Interdisziplinäre Kooperation zwischen Ethik und Recht». In: Settimio Monteverde (Hrsg.), Handbuch Pflegeethik. Stuttgart; 2012. S. $74 \mathrm{ff}$

7 Verordnung des EDI vom 29. September 1995 über Leistungen in der obligatorischen Krankenpflegeversicherung (KrankenpflegeLeistungsverordnung, KLV) 832.112.31. Erfahrung gar nicht in der

Damit widerspricht das KVG frontal den kantonalen Gesundheitsgesetzen. Den Pflegefachpersonen wird zwar ein eigenständiger Bereich zugestanden, die entsprechenden Leistungen von den Krankenkassen aber nicht übernommen - es sei denn, es liege eine ärztliche Verordnung vor ...

Wie lässt sich diese absonderliche Regelung erklären - und wie gefährlich wirkt sie sich haftungsrechtlich aus? Zu dieser gesetzgeberischen Fehlkonstruktion hat neben dem erwähnten, klischierten Bild der Pflege die nicht weniger naive Vorstellung des Arztes als «Gatekeeper», als neutraler und wissender Richter über Art und Umfang der erforderlichen Pflegeleistungen geführt. Mit dem Verordnungszwang für Pflegeleistungen wird der Arzt allerdings überfordert. Er muss Leistungen gegenzeichnen, für die er nicht kompetent ist - eben weil sie zum eigenverantwortlichen Bereich eines anderen Berufes gehören. Man kann nicht «halb eigenverantwortlich» sein. Die geltende Gesetzeslage stiftet mit dem Konstrukt einer «eigenverantwortlichen Hilfsperson» verhängnisvolle Verwirrung.

\section{Wer haftet wofür?}

Fangen wir mit dem ärztlich delegierten Bereich an: Bei Einhaltung der Delegationsgrundsätze geht die zivil-, straf- und disziplinarrechtliche Haftung für die Durchführung der delegierten Tätigkeit vollumfänglich auf die Pflegefachperson über. Begeht sie dabei einen Fehler, haftet sie jetzt schon alleine [3] ärztliche Anordnung oder Unterschrift hin oder her.

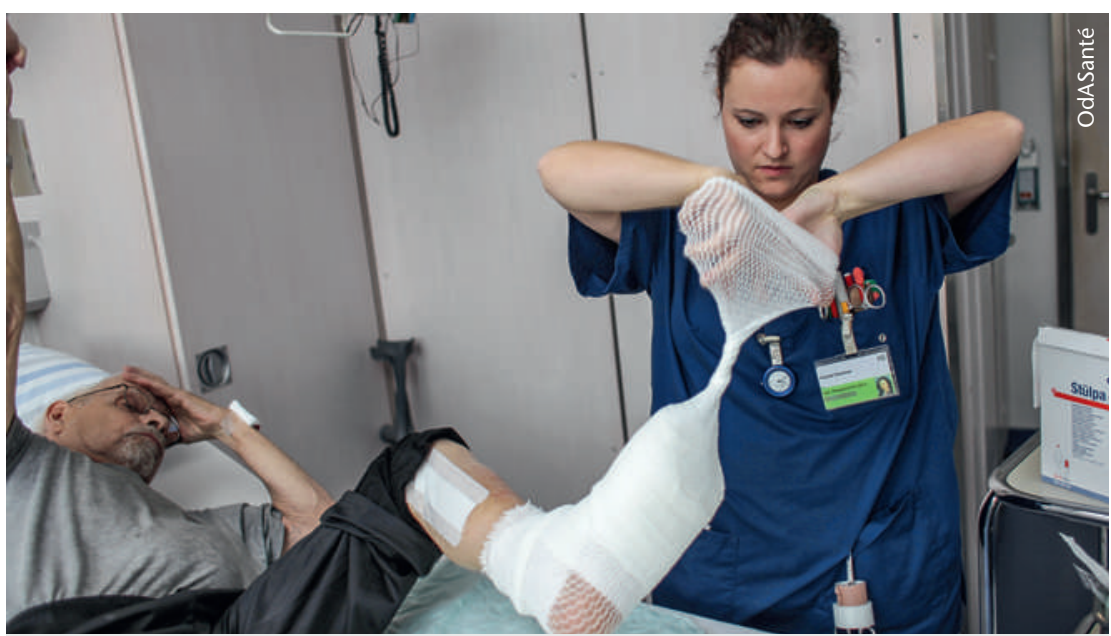

Schon jetzt gibt es Leistungen, die Pflegefachpersonen in voller eigener rechtlicher und fachlicher Verantwortung erbringen dürfen.
Dies gilt umso mehr da, wo sich die Pflegefachperson innerhalb ihres ureigenen, eigenverantwortlichen Kompetenzbereichs bewegt. Mit der vom KVG geforderten Unterschrift übernimmt der Arzt weder zivil-, straf- noch disziplinarrechtlich die Haftung für die Ausführung dieser Leistung.

Sie entspricht einer rein versicherungsrechtlichen Vorschrift, deren Zweck sich in der Auslösung einer Krankenversicherungsleistung erschöpft: Er bürgt dafür, dass die Leistung den krankenversicherungsrechtlichen Anforderungen entspricht - was er, aus den erwähnten Gründen, gar nicht kann.

\section{Unwirtschaftlicher Widersinn}

Wie absurd dies ist, belegt der Berufsalltag der freiberuflichen Pflegefachleute, in dem es sich oft genau umgekehrt verhält: Es wird nämlich von ihnen und nicht vom verordnenden Arzt - erwartet, über die Wirtschaftlichkeit, Zweckmässigkeit und Wirksamkeit von Massnahmen der Behandlungspflege Rechenschaft abzulegen!

Dass es sich bei der Unterschrift eines Arztes unter eigenverantwortlichen Pflegemassnahmen um einen (erst noch kostspieligen) Widersinn handelt, haben die verständigeren Krankenkassen erfasst [4], die verständigeren Ärzte sowieso [5]. Abgesehen davon wirkt sich nichts toxischer und hinderlicher auf eine gute interprofessionelle Zusammenarbeit aus als ein Auseinanderklaffen von Kompetenz und Verantwortung [6]. Die parlamentarische Initiative Joder will nichts mehr und nichts weniger, als die Verantwortung der Pflegefachpersonen in Übereinstimmung zu bringen mit ihren Kompetenzen - und das Gesetz in Übereinstimmung mit der Realität des Berufs.

\section{Die Katze im Sack?}

Eine weitere angstbesetzte Frage ist, ob denn klar sei, welche Tätigkeiten zum eigen- und welche zum ärztlich-delegierten Bereich der Pflege gehören, zumal die Initiative sehr offen festhält, die Abgrenzung obliege dem Bundesrat. Die Antwort ist auch hier schnell gegeben: Ja, es ist sehr klar. Art. 7 der sog. Leistungsverordnung zum KVG [7], der die von der Grundversicherung zu übernehmenden Pflegeleistungen definiert, unterscheidet explizit zwischen Massnahmen der Untersuchung und der Behandlung (anders ausgedrückt: der Diagnostik und der Therapie) einerseits, und Massnahmen der Abklärung, Beratung, Koordination und Grundpflege andererseits. Erstere, die den mitverantwortlichen Bereich abdecken, sind nach einhelliger Lehre und Rechtsprechung erschöpfend aufgelistet.

Der Angst, mit der Initiative «die Katze im Sack zu kaufen», kann also mit dem schlichten Verweis auf die Gesetzessystematik des KVG begegnet werden: Die versicherten Leistungen werden prinzipiell auf Verordnungsebene umschrieben und aufgezählt. Es kann der Initiative schlecht zum Vorwurf gemacht werden, dass sie sich an das bestehende System des KVG hält. 